\title{
A Real Time Sign Language Recognition System using Hand Tracking
}

\author{
Mrs Smitha P ${ }^{* 1}$, Bhuvanashree R ${ }^{* 2}$, Jyothi K.N*3, Sagar Tandure ${ }^{* 4}$ \\ ${ }^{* 1}$ Assistant Professor, Department of Information Science and Engineering, \\ East West Institute of Technology, Bengaluru, Karnataka, India. \\ ${ }^{* 2,3,4}$ Student, VTU, Department of Information Science and Engineering, \\ East West Institute of Technology, Bengaluru, Karnataka, India.
}

\begin{abstract}
While the majority of individuals can communicate using spoken language, certain persons with restricted skills must communicate using sign language, which involves hand and finger gestures, even if not everyone can comprehend what they are saying. The fast advancement ofinformation technology can aid in the comprehension and translation of gestures. Using the Kinect camera, this project seeks to develop and build a system that recognises alphabet sign language gestures with the feature of fingers opening. The tracking process and the recognitionprocess are the two steps of the recognition system. The tracking procedure was completed by using the hands tracking approach. The recognition process is divided into two phases: the first is to get input that will be used as reference data and data testers, which is accomplished by calculating vectors and vector angles; the second is to recognise gestures, which is accomplished by calculating the Euclid distance. The system can detect motions alphabets withan average degree of accuracy of 69.79 percent, which varies according on the scenario.
\end{abstract}

\section{INTRODUCTION}

Today, robots are utilised successfully in a variety of fields, including industrial manufacturing,military operations, deep sea drilling, and space research. This achievement has piqued curiosity in the potential of utilising robots in human social settings, particularly in the care of the elderly and disabled. Humans communicate effortlessly and spontaneously in social situations using both speech (audio) and gesture (visual) without the use of any external technologies (such as keyboards) that require specific training. The goal of this project is to use basic hand gestures to operate a robot. The fundamental motive is the desire to create robots that can interact with humans naturally without the use of any special technologies.

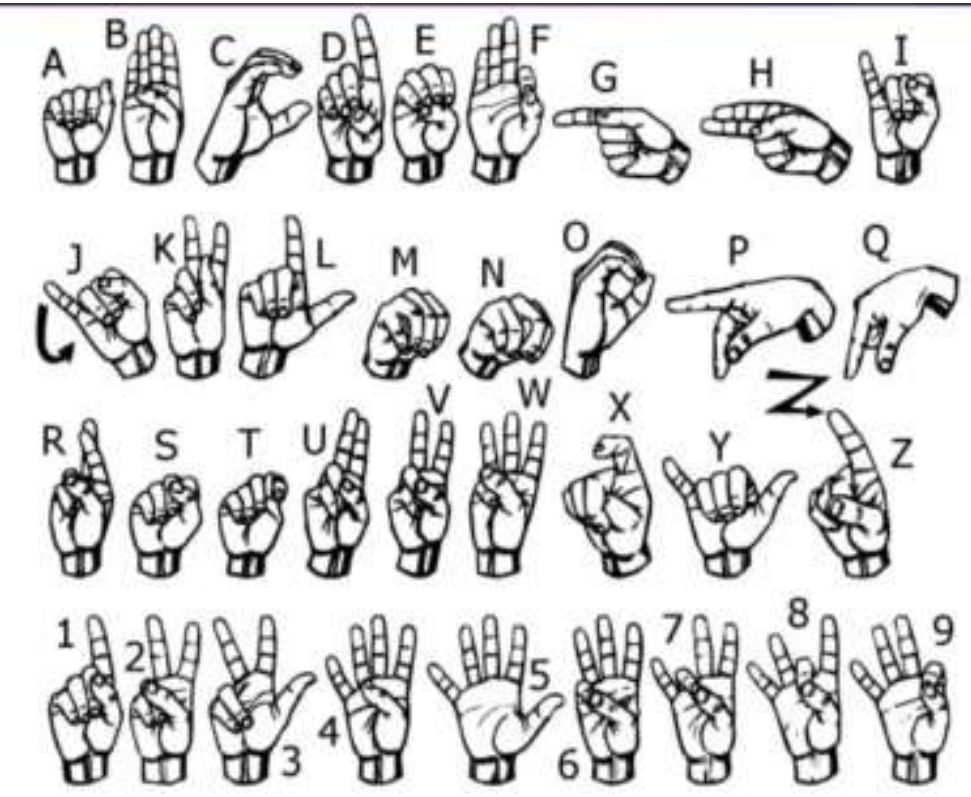


International Journal of Innovative Research in Electrical, Electronics, Instrumentation and Control Engineering

Vol. 9, Issue 7, July 2021

DOI 10.17148/IJIREEICE.2021.9719

\section{LITERATURE SURVEY SUMMARY}

Russell and Norvig [6] described three general methodologies used in computer vision to extractmeaningful information from visual input. The first is the feature extraction strategy, which focuses on applying simple computations directly to digital images to quantify some usable attribute, such as size. This is based on well-known image processing algorithms for noise reduction, filtering, object detection, edge detection, texture analysis, optical flow calculation, and segmentation, all of which are routinely used to prepare images for further study. This is also regarded as a "uninformed" strategy.

The second strategy is the recognition approach, which focuses on identifying and labelling things based on knowledge of properties that groups of similar items have, such as shape or appearance, or patterns of elements, which are sufficient to define classes. In this case, computer vision employs artificial intelligence techniques in knowledge representation to allowa "classifier" to match objects to classes based on the pattern of their characteristics or structural descriptions. A classifier must "learn" the patterns by being fed a training set of objects and their classes, with the goal of minimising errors and maximising successes via a step-by-step improvement process.

The third method is the reconstruction method, which focuses on creating a geometric model ofthe environment based on the picture or images as a starting point for action. This relates to thepicture understanding step, which is the most advanced and difficult phase of computer vision processing. The focus here is on allowing the computer vision system to build internal models based on the data provided by the pictures, and then reject or update these internal models when they are validated against the real world or other criteria. Image comprehension occurs when the internal model is compatible with the real environment.

\section{PROPOSED WORK}

Our proposed system uses ASL, which is a sign language based on hand movements that is utilised by deaf people. Another need we have is that the system be in the form of a wearable device with its own data collecting module, which maps the data to the relevant sign, and then converts the sign to the alphabet - all in one. To complete the task, we did not utilise a separateprocessing unit or a large CPU running a Matlab application.

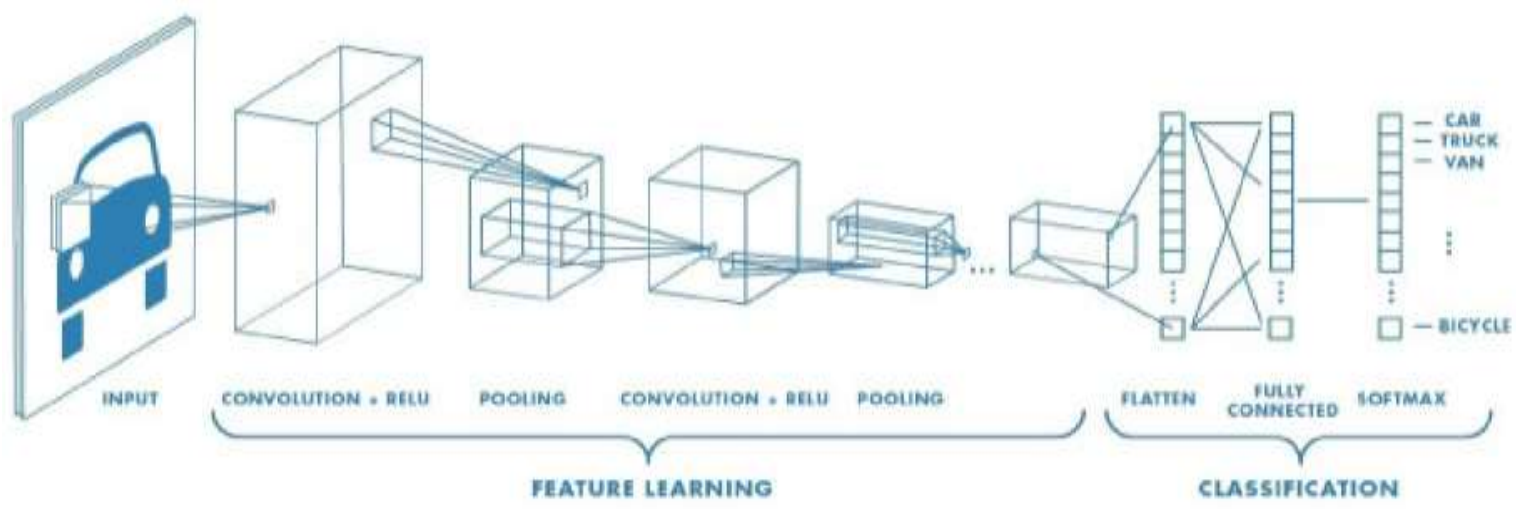

\section{OBJECTIVES}

This project's major goal is to make a contribution to the field of automatic sign language recognition. The identification of static sign language movements is the subject of this study.Deep learning was utilised to recognise 24 alphabets and 09 numerals in this study. We developed a convolutional neural network classifier capable of accurately recognising static sign language movements. The results demonstrate that when we add more data from variousparticipants during training, accuracy increases. We've also made a basic graphical user interface (GUI) application to test our classifier.

\section{CNN (CONVOLUTIONAL NEURAL NETWORKS)}

\section{ALGORITHM:}

CNN (Convolutional Neural Networks) make image processing computationally manageable by filtering connections by proximity. Rather of connecting every input to every neuron in a layer, CNN purposefully limits the connections such that each neuron only takes information from a limited subset of the layer. As a result, each neuron is solely responsible 
International Journal of Innovative Research in Electrical, Electronics, Instrumentation and Control Engineering

Vol. 9, Issue 7, July 2021

\section{DOI 10.17148/IJIREEICE.2021.9719}

forprocessing a part of a picture. As a result, by eliminating many of these insignificant connections, CNN eliminates the problem of increased computational burden. It is the most effective algorithm for picture recognition.

\section{RECOGNITION OF HAND GESTURE}

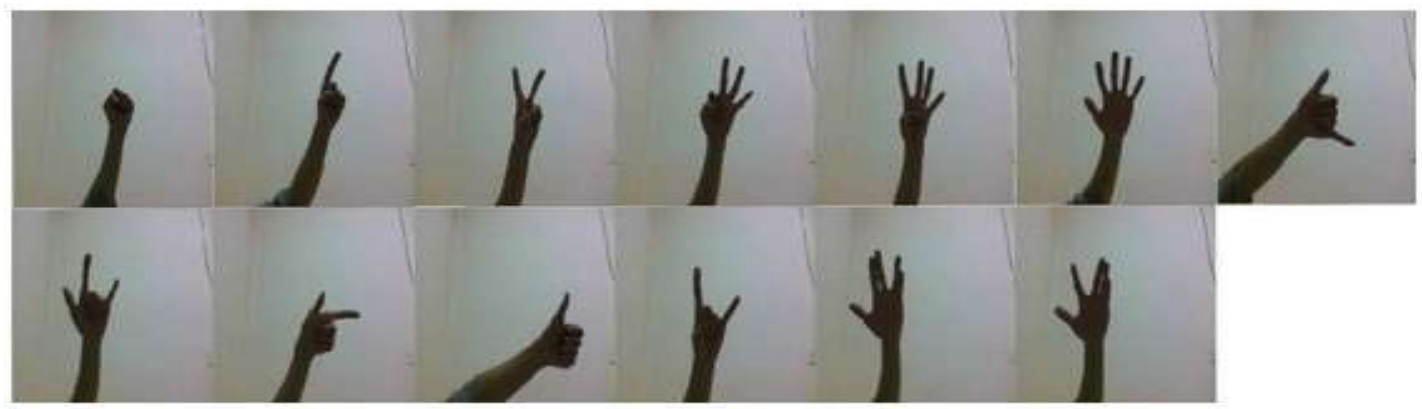

When the fingers are detected and recognized, the hand gesture can be recognized using a simple rule classifier. In the rule classifier, the hand gesture is predicted according to the number and content of fingers detected. The content of the fingers means what fingers are detected. The rule classifier is very effective and efficient. For example, if three fingers, that is, the middle finger, the ring finger, and the little finger, are detected, the hand gesture is classified.

\section{CONCLUSION}

This study introduces a novel approach for hand gesture recognition. The background subtraction approach is used to detect the hand region from the backdrop. The palm and fingers are segmented after that. The fingers in the hand picture are found and recognised based on the segmentation. Hand gesture recognition is achieved using a simple rule classifier. On a data set of many hand pictures, the performance of our approach is assessed. The results of the experiments demonstrate that our method works effectively and is suitable for real-time applications. Furthermore, using an image collection of hand motions, the suggested approach beats the state-of-the-art FEMD.

The suggested method's efficacy is strongly dependent on the results of hand detection. If thereare moving objects with a color that is close to that of the skin, the items are detected as a consequence of the hand detection and decrease the hand gesture recognition performance.

Machine learning algorithms, on the other hand, can distinguish the hand from the backdrop. ToF cameras give depth information that can help enhance hand detection accuracy. To solvethe complicated backdrop problem and increase the robustness of hand identification, machinelearning algorithms and ToF cameras may be employed in future research.

\section{REFERENCES}

[1]. Automatic Sign Language Finger Spelling Using Convolution Neural Network:AnalysisISSN: 1311-8080 (printed version); ISSN: $1314-3395$ (online version)

[2]. Transactions on medical imaging transfer learning improve supervised image segmentation across imaging protocols. 0278-0062 (c) 2013 IEEE

[3]. Using vaes and normalizing flows for one-shot text-to-speech synthesis of expressive speech. 978-1-5090-6631-5/20/\$31.00 @2020 IEEE

[4]. An Overview of Text-to-Speech Synthesis. 0-7803-64 16-3/00/\$10.0002000 • IEEE Development of GUI for Text-to-Speech Recognition using Natural Language Processing. 978-1-5386-5550-4/18/\$31.00 @2018 IEEE SSVM Classifier and Hand Gesture based Sign Language Recognition. 978-1-5386-6483-4/18/\$31.00 @2018 IEEE

[5]. Detecting hand-palm orientation and hand shapes for sign language gesture recognition using 3d images. 978-1-4673-5600-8/12/\$31.00 @2012 IEEE Devanagari Printed Text to Speech Conversion using OCR. 978-1-5386-1442-6/18/\$31.00 @2018 IEEE

[6]. A Realtime Alphabets Sign Language Recognition System Hands Tracking. 978-1-5090-5548-7/16/\$31.00 @2016 IEEE https://www.kdnuggets.com/2017/08/convolutional-neural-networks-imagerecognition.html 